\title{
Physics at the Compact Linear Collider
}

\author{
I. Božović-Jelisavčić ${ }^{1) *}$ \\ On behalf of the CLICdp collaboration \\ ${ }^{*}$ Vinca Institute of Nuclear Sciences, University of Belgrade, Serbia
}

\begin{abstract}
This paper (based on an invited talk at the $18^{\text {th }}$ Lomonosov Conference on Elementary Particle Physics) provides an overview of the physics program at CLIC, including updates on the ongoing studies on t-quark precision observables, massive vector-boson scattering and di-photon processes at high energies.
\end{abstract}

Talk presented at the $18^{\text {th }}$ Lomonosov Conference of Elementary Particle Physics, Moscow, 24-30 August, 2017,- special issue of the International Journal of Modern Physics A.

\footnotetext{
1) ibozovic@ vinca.rs
} 


\title{
Physics at the Compact Linear Collider
}

\author{
Ivanka Božović-Jelisavčića on behalf of the CLICdp collaboration \\ Vinca Institute of Nuclear Sciences, University of Belgrade 11000 Belgrade, Serbia \\ Abstract. This paper (based on an invited talk at the $18^{\text {th }}$ Lomonosov Conference on Elementary \\ Particle Physics) provides an overview of the physics program at CLIC, including updates on \\ the ongoing studies on t-quark precision observables, massive vector-boson scattering and di- \\ photon processes at high energies.
}

\section{Introduction}

Highlights from the topical studies ongoing at CLIC in the Higgs, top and Beyond the Standard Model (BSM) sectors are given to illustrate the capabilities of CLIC to address relevant questions in the post-LHC era. An energy-staged approach that includes three running center-of-mass energies $(380 \mathrm{GeV}, 1.4 \mathrm{TeV}$ and $3 \mathrm{TeV})$ is optimized to the physics program, including the CLIC unique sensitivity at the highest center-of-mass energy to a possible extensions of the Standard Model (SM). With its full statistics of $\sim 10^{6}$ Higgs bosons, crucial measurements of the Higgs properties will be possible to indicate the validity scale of the Standard Model.

As at other $e^{+} e^{-}$colliders, the CLIC experimental environment is practically free of QCD background and thus suitable for precision measurements. As discussed in Section 1.1, machine related background can be reduced to the satisfactory level for physics analyses. Staged implementation can provide a long-term precision physics program to complement the LHC searches.

\subsection{CLIC accelerator and detector}

The CLIC accelerator is based on a novel two-beam acceleration scheme, where a high-intensity beam (drive beam) is used to generate RF power to the main beam. Using normal-conducting accelerator structures, the two-beam accelerations provides gradients of $100 \mathrm{MV} / \mathrm{m}$ as has been demonstrated at the CTF3 test facility [1]. Short $\left(\sigma_{x}=40 \mathrm{~nm}, \sigma_{y}=1 \mathrm{~nm}\right)$ and dense $\left(\sim 10^{9}\right.$ particles $)$ bunches, lead to a strong beamstrahlung induced by the electromagnetic fields of the opposite bunches. Consequently, beamstrahlung photons convert to hadrons causing the occupancy of the central detectors. With a time-stamping window of $10 \mathrm{~ns}$ and appropriate cuts on the transverse momentum and reconstructed time of particles in the calorimeter, the average depositions from this type of background can be reduced to the acceptable level [2]. On the other hand, beamstrahlung severely deteriorates the luminosity spectrum, in particular at the highest center-of-mass energies. However, it has been shown in [3] that the effect can be controlled at a permille level in the peak region $(>80 \%$ of the nominal center-of-mass energy).

\footnotetext{
${ }^{a}$ E-mail: ibozovic@vinca.rs
} 
A new, post-CDR detector model (CLIC_det) has been proposed [4] with the consideration to the ultimate precision for physics. The detector performance is optimized to physics requirements for hermeticity, flavor tagging, jet energy resolution, $p_{T}$ reconstruction, etc. Detector comprise all-silicon tracking and compact calorimetry, placed in a 4T magnetic field. High-granularity calorimeters are optimized to the particle flow performance in order to enable a jet energy resolution of $\sim 3.5-5 \%$ for jet energies of $10 \mathrm{GeV}$ up to $1.5 \mathrm{TeV}$. Together with the flavor separation, the above is particularly relevant to distinguish between $Z, W$ or Higgs bosons in their hadronic decay modes.

\section{Higgs studies at CLIC}

\subsection{Combined fit of the Higgs measurements}

An extensive program of physics studies based on full simulations of detector and physics processes, together with a full event reconstruction, has been performed in the Higgs sector and documented in [5]. Higgsstrahlung and $W W$-fusion are dominant production mechanisms of the Higgs boson at low and high center-of-mass energies, respectively. Depending on the production mechanism, appropriate polarization could eventually double the statistics. A combined study of these two processes can be employed to probe the Higgs width and couplings in a model-independent way. This leads to a determination of the Higgs couplings at a level of $\sim 1 \%$, except for the rare decays to light particles such as muons or photons. Assuming that the Higgs total width is constrained by the SM decays, the statistical precision of the Higgs couplings can be improved to a sub-percent level. Both model-independent and modeldependent fits exploit the full statistics available at all CLIC energy stages in a cumulative way. Details of the combined fits can be found in [5] and have already been discussed in [6]. Model-dependent results allow for a comparison to the LHC and HL-LHC experiments where a similar approach is employed. To illustrate the CLIC sensitivity in comparison to the HL-LHC with the full statistics of $3000 \mathrm{fb}^{-1}$, results of the model-dependent fit are given in Figure 1 [7]. As can be seen, the CLIC will be able to provide comparable or better precision of the Higgs couplings than HL-LHC.

\subsection{Higgs self-coupling}

At the high center-of-mass energies of $1.4 \mathrm{TeV}$ and $3 \mathrm{TeV}$, a double-Higgs production can be used to measure the Higgs boson trilinear self-coupling parameter $\lambda$ that determines the shape of the Higgs potential. Several processes contribute to the double Higgs production, as illustrated in Figure 2 (left), where only one Feynman diagram (a) has the sensitivity to $\lambda$. Contribution of the non-sensitive Feynman diagrams to the double-Higgs production cross-section is taken into account with the parameterization of the double-Higgs production cross-section $\sigma\left(e^{+} e^{-} \rightarrow v_{e} \bar{v}_{e} H H\right)$ w.r.t. the 


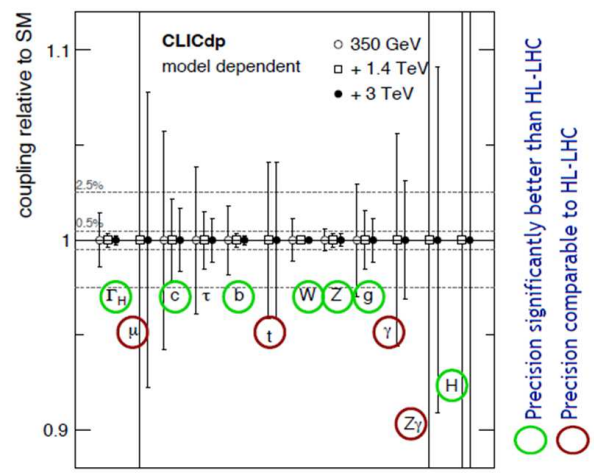

Figure 1: Higgs couplings relative statistical uncertainties in a model-dependent fit. The full statistics of $500 \mathrm{fb}^{-1}$ and $1500 \mathrm{fb}^{-1}$ and $2 \mathrm{ab}^{-1}$ is assumed for $350 \mathrm{GeV}, 1.4 \mathrm{TeV}$ and $3 \mathrm{TeV}$ CLIC, respectively, and $3000 \mathrm{fb}^{-1}$ for the HL-LHC.

self-coupling parameter $\lambda$ :

$$
\frac{\Delta \lambda}{\lambda} \sim \kappa \frac{\Delta\left|\sigma\left(H H v_{e} \bar{v}_{e}\right)\right|}{\sigma\left(H H v_{e} \bar{v}_{e}\right)}
$$

Simulation at the generator level gives values of 1.22 and 1.47 for the scaling factor $\kappa$, at $1.4 \mathrm{TeV}$ and $3 \mathrm{TeV}$ center-of-mass energy, respectively [5]. Two signal final states are considered at both energies: $H H \rightarrow b \bar{b} b \bar{b}$ and $H H \rightarrow b \bar{b} W^{+} W^{-}$, together with a complete list of relevant background processes. Signal selection ensures that there is no overlap between the different final states of the signal, while a multivariate analysis is employed to separate each signal from background processes.

Combined results for the both Higgs decay channels, including electron polarization of $-80 \%$, leads to the relative statistical uncertainty of $\lambda$ of $19 \%$ and $16 \%$ at 1.4 and $3 \mathrm{TeV}$ respectively [8]. The achievable precision of $\lambda$ can be improved further if multivariate analysis method would be employed to separate between $\lambda$-sensitive and non-sensitive double-Higgs production channels.

\section{Top physics at CLIC}

With its energy-staged implementation, CLIC offers a broad program of measurements to study the top-quark. The above includes the top mass determination from a threshold scan with the relative statistical uncertainty of $\sim 15 \mathrm{MeV}$ [9], direct access to the top Yukawa coupling at energies above $500 \mathrm{GeV}$ and the precision studies of the top electroweak (EW) observables providing sensitivity to BSM scenarios. Excellent flavor tagging and jet reconstruction capabilities at CLIC enables precision 

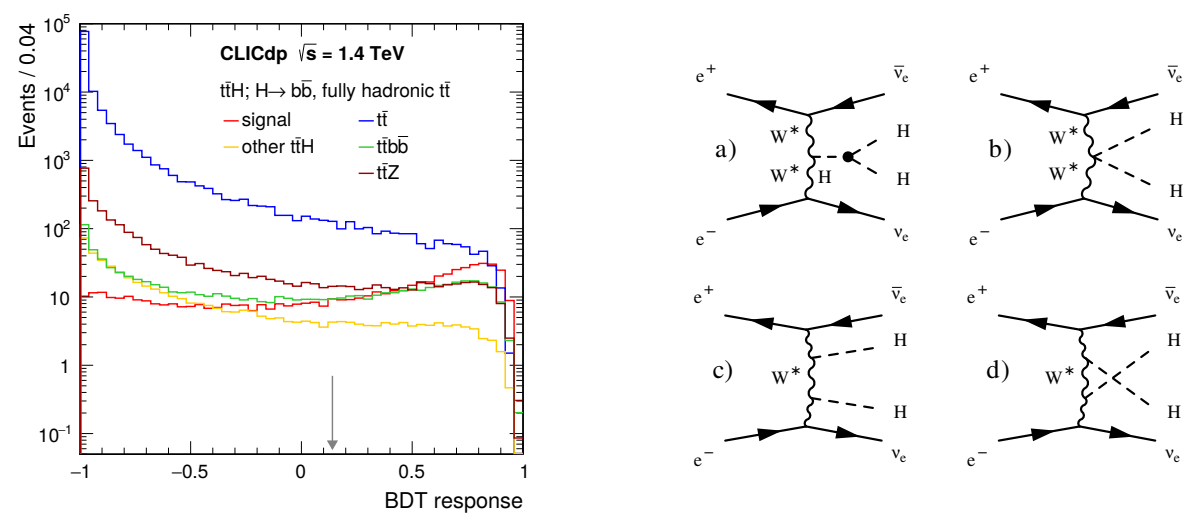

Figure 2: Left: Dominating Feynman diagrams for double-Higgs production at CLIC center-of-mass energies above $1 \mathrm{TeV}$, where only diagram (a) has a sensitivity to the Higgs self-coupling $\lambda$. Right: BDT based separation of the fully hadronic $t \bar{t} H$ signal from various background processes. The arrow denotes the BDT output value corresponding to the maximal significance.

top Yukawa coupling measurement at $1.4 \mathrm{TeV}$ center-of-mass energy. The relative statistical uncertainty of the $t \bar{t} H$ production cross-section $\sigma$ translates into a relative statistical uncertainty of the top Yukawa coupling $y_{t}$ :

$$
\frac{\Delta y_{t}}{y_{t}}=0.53 \frac{\Delta \sigma}{\sigma},
$$

leading to a top Yukawa relative statistical uncertainty of $\sim 4 \%$, assuming electron polarization of $-80 \%$ [5]. Analysis has been performed in the Higgs dominant decay channel to $b \bar{b}$, while $W$ bosons from the top-quark decays can either decay leptonicly or hadronically, leading to 6 to 8 -jet topology of the final state. The scaling factor in Eq. 2 is determined at the generator level, assuming a Standard Model value of the top Yukawa coupling. Illustration of the signal separation from various background processes, based on the multivariate method (BDT) output value, is given in Figure 2 , right [5]. Multivariate approach allows to maximize a statistical significance of the signal in the presence of various physics backgrounds.

\subsection{Top-quark precision EW observables}

Top-pair production through the $Z, \gamma$ exchange in the s-channel, provides direct access to the top EW couplings sensitive to a higher order corrections from BSM processes. The coupling associated to the top- $Z(\gamma)$ vertices is defined through a set of CP conserving and $\mathrm{CP}$ violating form factors that can be constrained through measurements of the sensitive EW observables (cross-section, forward-backward asymmetry, helicity angle distribution in top decays). Beam polarization allows to access different 

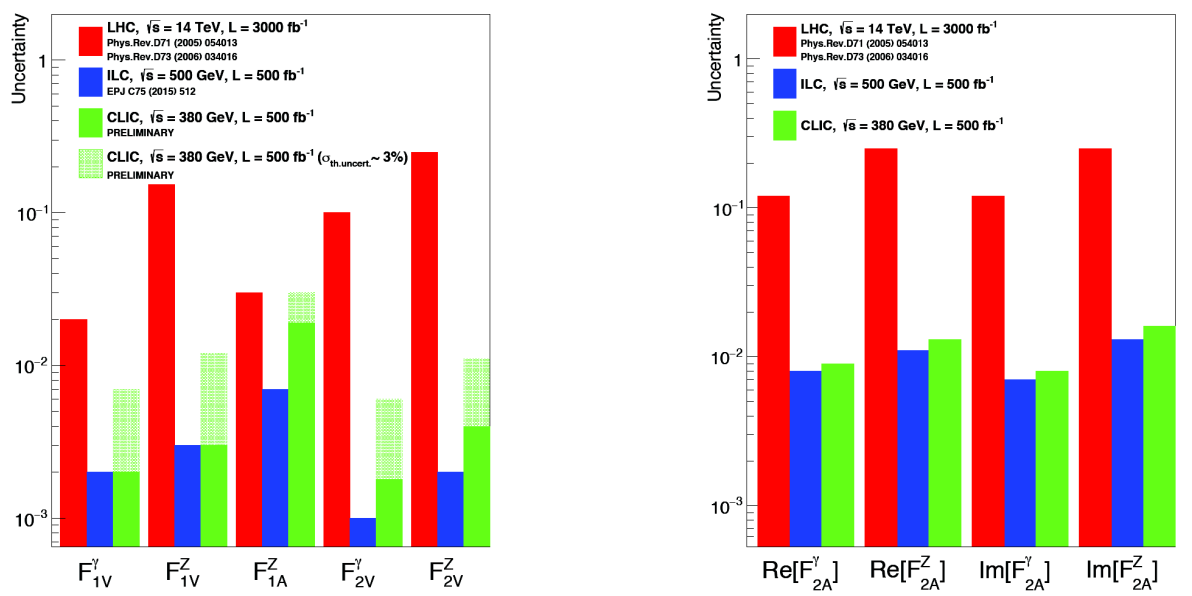

Figure 3: Expected precision on the top-quark EW form factors at different experiments.

form factors independently. Figure 3[9] gives the relative statistical precision of the top-quark $\mathrm{CP}$ conserving and $\mathrm{CP}$ violating form factors at different experiments. It demonstrates that future electron-positron colliders (in example of ILC and CLIC) are powerful instruments to study Higgs $\mathrm{CP}$ properties.

\section{BSM searches}

Potential signatures of BSM physics can be searched for at CLIC either through direct reconstruction of new particles, with an approximate mass reach of $\sqrt{s} / 2$, or indirectly through the possible deviations in precision observables (cross sections, asymmetries, couplings, etc.). Indirect searches extend the sensitivity for BSM physics beyond the direct kinematic reach of the machine, up to (model-dependent) scales of several tens of TeV. In this paper, two examples of novel CLIC studies are given in Sections 4.1 and 4.2 to illustrate the possible constraints on BSM physics through indirect searches.

\subsection{Massive vector boson scattering at high energies}

Massive vector boson scattering, and $W^{+} W^{-}$scattering in particular, are sensitive probes to the higher-dimension operators in the Effective Field Theory (EFT) approach. Such a scattering process $W^{+} W^{-} \rightarrow V V$, with $V=W, Z$ is illustrated in Figure 4, left. Anomalous couplings at the quartic vertex (i.e. $\alpha_{4}, \alpha_{5} \neq 0$ ) result in modification of the sensitive observables like the cross-section and invariant mass of the $V V$ system. Fully hadronic final state of the $V V$ system is separated from the background processes in a multivariate analysis approach. A chi squared $\left(\chi^{2}\right)$ fit is employed to extract the couplings $\alpha_{4}$ and $\alpha_{5}$, and the corresponding confidence inter- 

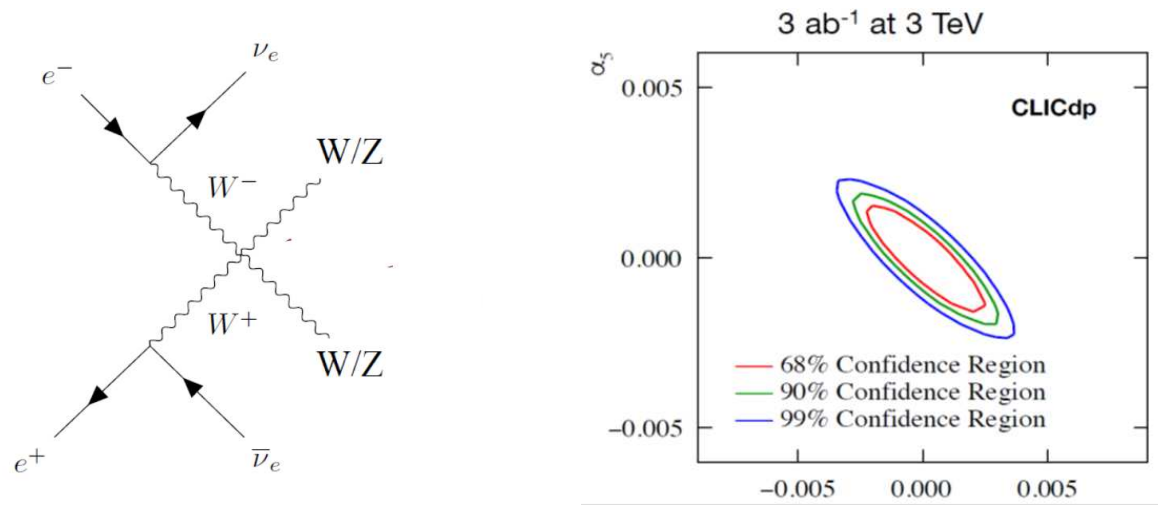

Figure 4: Left: Feynman diagram for $W^{+} W^{-}$scattering. Right: Confidence intervals for the quartic couplings $\alpha_{4}$ and $\alpha_{5}$, simulated at 3 TeV CLIC with $3 \mathrm{ab}^{-1}$ of the integrated luminosity.

vals have been derived. Due to a larger statistics, coupling determination precision at $3 \mathrm{TeV}$ CLIC (Figure 4, right) is improved for an order of magnitude in comparison to the measurement at $1.4 \mathrm{TeV}$ center-of-mass energy [8]. As for the top-physics studies, this measurement also relies on the excellent jet reconstruction.

\subsection{Di-photon production}

Di-photon production is a well described QED process that can be exploited to search for the possible deviations of the sensitive observables (cross-section, photon polar angle distribution) from the Standard Model predictions. Depending on the relative uncertainty of the integrated luminosity, various BSM models can be probed at a kinematic limit 10-20 times higher than at LEP. This is illustrated in Table 1 [8]. Forward electron tagging is crucial for this measurement in order to suppress background from the Bhabha scattering.

Table 1: $95 \%$ CL exclusion limits for various BSM models, obtained from di-photon production at $3 \mathrm{TeV}$ CLIC with $2 \mathrm{ab}^{-1}$ and at LEP.

\begin{tabular}{lllll}
\hline Scenario & $\Delta L=0.2 \%$ & $\Delta L=0.5 \%$ & $\Delta L=0.1 \%$ & LEP limit \\
\hline $\begin{array}{l}\text { QED cut-off (finite elec- } \\
\text { tron size) } \Lambda_{Q E D}\end{array}$ & $6.52 \mathrm{TeV}$ & $6.33 \mathrm{TeV}$ & $6.01 \mathrm{TeV}$ & $\sim 390 \mathrm{GeV}$ \\
Contact interactions $\Lambda^{\prime}$ & $20.7 \mathrm{TeV}$ & $20.1 \mathrm{TeV}$ & $18.9 \mathrm{TeV}$ & $\sim 830 \mathrm{GeV}$ \\
Extra dimensions & $16.3 \mathrm{TeV}$ & $15.9 \mathrm{TeV}$ & $15.3 \mathrm{TeV}$ & $\sim 1 \mathrm{TeV}$ \\
$M_{S} / \Lambda^{1 / 4 \quad}$ & $5.03 \mathrm{TeV}$ & $4.87 \mathrm{TeV}$ & $4.7 \mathrm{TeV}$ & $\sim 250 \mathrm{GeV}$ \\
Excited electron $M_{e^{+}}$ &
\end{tabular}




\section{Summary}

CLIC is a mature future linear collider option with the physics program combining precision measurements with direct and indirect discovery potential. The CLIC detector model is being optimized to meet the performance requirements from the physics studies, in terms of multi-jet final state reconstruction, photon reconstruction, missingmomentum measurement, forward electron tagging and many more. Staged implementation results in a broad physics program, from precision studies of the Higgs and top-quark physics to BSM probes. At the lowest center-of-mass energy, CLIC features model independence in the Higgs coupling measurements, achievable with a statistical precision at the percent level for most of the couplings. Operation at high energies enables precision measurement of rare Higgs decays, Higgs self-coupling, Higgs mass and CP properties. BSM measurements also benefits from the high-energy operation, with the sensitivity extended far beyond the available center-of-mass energy. Topquark measurements serve as additional probe to the EW and BSM sectors.

\section{Acknowledgments}

Vinca Institute activity at CLIC/CLICdp has been supported by the Ministry of Education, Science and Technological Development of the Republic of Serbia, through the project OI171012.

[1] L. Linssen et al. (editors), Physics and Detectors at CLIC: CLIC Conceptual Design Report, ANL-HEP-TR-12-01, CERN-2012-003, DESY 12-008, KEK Report 2011-7, CERN, 2012.

[2] H. Abramowicz et al., Physics at the CLIC e+e- Linear Collider Input to the Snowmass Process 2013, 2013, arXiv:1307.5288.

[3] S. Lukic, I. Bozovic Jelisavcic, M. Pandurovic, I. Smiljanic, Correction of beam-beam effects in luminosity measurement in the forward region at CLIC, JINST 8 (2013) P05008.

[4] M. Weber, The CLIC detector, EPS-HEP 2017, July, 2017, Venice, Italy.

[5] H. Abramowicz et al. CLICdp Collaboration, Higgs physics at the CLIC electron-positron linear collider, Eur.Phys.J. C77 (2017), 475.

[6] I. Bozovic Jelisavcic, CLIC Physics Overview, in the Proceedings of the 17th Lomonosov Conference on Elementary Particle Physics, August 20-26, 2015, Moscow, Russia, Ed. by A. Studenikin, World Scientific, Singapore.

[7] V. Martin, Status of CLIC, $28^{\text {th }}$ International Symposium on Lepton Photon Interactions at High Energy, August, 2017, Guangzhou, China.

[8] F. Simon, CLIC Analyses Highlights, AWLC 2017, June, 2017, Stanford, USA.

[9] A. Filip Zarnecki, Top physics at CLIC and ILC, PoS ICHEP2016 (2016), C16-08-03 